Revue d'histoire de l'Amérique française

ZRS REVUE D.HISTOIRE DE L'AMÉRIQUE FRANÇAISE

\title{
L'idée de fédération chez Étienne Parent, 1831-1852
}

\section{Louis Nourry}

Volume 26, numéro 4, mars 1973

URI : https://id.erudit.org/iderudit/303211ar

DOI : https://doi.org/10.7202/303211ar

Aller au sommaire du numéro

Éditeur(s)

Institut d'histoire de l'Amérique française

ISSN

0035-2357 (imprimé)

1492-1383 (numérique)

Découvrir la revue

Citer cet article

Nourry, L. (1973). L’idée de fédération chez Étienne Parent, 1831-1852. Revue

d'histoire de l'Amérique française, 26(4), 533-557.

https://doi.org/10.7202/303211ar d'utilisation que vous pouvez consulter en ligne.

https://apropos.erudit.org/fr/usagers/politique-dutilisation/ 


\section{L'IDÉE DE FÉDÉRATION CHEZ ÉTIENNE PARENT}

\section{1-1852}

LOUIS NOURRY

Département d'histoire

Université de Montréal

$\mathrm{Au}$ début du $\mathrm{XIX}^{\mathrm{e}}$ siècle, la petite bourgeoisie professionnelle ${ }^{1}$ supplante l'aristocratie cléricale à la tête de la société rurale canadienne et accapare le leadership à la Chambre d'assemblée. De plus, à partir de 1820, lorsque "des difficultés viennent troubler l'agriculture canadienne dans ses trois grands facteurs internes: marché, technique et territoire" 2 , elle canalise l'inimitié de l'habitant, pour qui "l'Anglais fait alors figure d'objet de ses craintes et de source de ses difficultés", ${ }^{3}$ dans le combat qu'elle mène pour déloger du pouvoir la bourgeoisie marchande et les hauts fonctionnaires britanniques installés dans les conseils.

Ce conflit atteint son sommet en 1827. La Chambre d'assemblée refusant de voter les subsides sous une forme permanente, Dalhousie proroge, le 7 mars, la session ouverte le 23 janvier et, en juillet, dissout le douzième Parlement. Les élections au cours desquelles "fut employé pour la première fois le mot patriote pour désigner le parti canadien" 4 reportent la même députation au Parlement. Le 20 novembre, le gouverneur refuse d'entériner le choix de Papineau comme "orateur" de la Chambre et il clôt la session deux jours plus tard, lorsque les représentants du peuple lui contestent le droit de leur demander de modifier leur décision. Les députés en appellent à leurs électeurs. On rédige des pétitions. Pour les appuyer à Londres, les Canadiens délèguent John Neilson, Augustin Cuvillier et Denis-Benjamin Viger. Les Britanniques leur opposent Samuel Gale.

1 Gilles Bourque, Classes sociales et question nationale au Québec, 1760-1840 (Montréal, 1970), 161-179.

2 Maurice Séguin, La "Nation canadienne" et l'agriculture, 1760-1850 (Trois-Rivières, 1970), 224.

3 Fernand Ouellet, "Le nationalisme canadien-français: De ses origines à l'insurrection de 1837", The Canadian Historical Review (University of Toronto Press), XLV (1964) : 288.

${ }_{4}$ Gérard Filteau, Histoire des Patriotes (Montréal, 1938), 1:129.

[ 533 ]

RHAF, vol 26, no 4 (mars 1973) 
Au printemps de 1828, le secrétaire d'Etat aux colonies, Huskisson, fait accepter par la Chambre des Communes la création d'un comité chargé d'enquêter sur la situation au Canada et de faire des recommandations. Canadiens et Britanniques s'affrontent. ${ }^{5}$ Ceux-ci réclament la colonisation intégrale de la vallée du Saint-Laurent et l'anglicisation progressive des Canadiens qui, de leur côté, requièrent un fonctionnement harmonieux de la Constitution de 1791 et une évolution des structures impériales vers l'octroi d'une plus grande autonomie aux colonies sur le plan intérieur. Le Rapport que le comité dépose le 22 juillet n'avance que de grands principes de conciliation; il ne provoque aucun débat et n'est pas mis aux voix. Dalhousie est toutefois rappelé. Son successeur, Sir James Kempt, ne parvient pas à concilier le parti patriote et le parti anglais qui, lors des élections de 1830, remportent respectivement 60 et 24 sièges. Après le départ de Kempt, Lor:d Aylmer convoque le quatorzième Parlement pour le 25 janvier 1831. Lorsqu'il annonce l'abandon de tous les revenus, à l'exception du revenu casuel et territorial (7,000 livres par an), en échange d'une liste civile de 19,500 livres votées pour la vie du roi, la Chambre rejette ce compromis et ne vote que des appropriations temporaires. Le 31 mars, le gouverneur met un terme à la session.

Le parti patriote durcit ses positions quand il voit son affirmation nationaliste s'inscrire dans le "synchronisme historique". ${ }^{6}$ Depuis 1828 les Etats-Unis vivent à l'heure de la démocratie jacksonienne, en Angleterre, l'aile radicale du parti libéral, qui vient d'accéder au pouvoir sous la direction de lord Charles Grey, préconise l'affranchissement des colonies de la tutelle métropolitaine et O'Connell agite la question du retour au "Home Rule" pour l'Irlande. Sur le continent européen, "il existe, dans certains milieux, une mystique, une 'religion' de la liberté, qui apparaît comme la condition nécessaire du progrès de l'humanité". 7 En février 1830, la Grèce s'émancipe. La révolution parisienne de Juillet consacre la chute de la dynastie que la volonté des Alliés avait restaurée en 1814. Le 4 octobre, la Déclaration d'indépendance des Flamands et des Wallons qui se sont unis contre les Hollandais à la suite de la formation d'un senti-

${ }^{5}$ Louise Lacour-Brossard, L'enquête impériale de 1828: Témoin de l'affrontement des deux nationalités au Bas-Canada, thèse de M.A., [Histoire] (Université de Montréal, 1971), 141 pages.

6 Lionel Groulx, Histoire du Canada français depuis la découverte (Montréal et Paris, 1965), 2:206.

7 Pierre Renouvin, Histoire des relations internationales, Le XIXe siècle, Première partie, De 1815 à 1851 (Paris, 1954), 5:11. 
ment national belge, devient effective. La Pologne s'insurge contre le tsar et proclame sa liberté le 25 janvier 1831 .

Dans ce contexte, Etienne Parent, admis au barreau en 1829 à l'âge de 27 ans, ressuscite Le Canadien de Québec le 7 mai 1831, après six ans d'éclipse. Dès lors lui incombe un rôle de penseur, d'éducateur et de guide. Etonnamment prolifique, il aborde tous les domaines de l'agir collectif, transmet toute la tradition orale et écrite, observe et analyse les réactions de la minorité britannique à laquelle il livre une lutte soutenue. Il façonne et polarise les aspirations canadiennes. Se plaçant à l'avant-garde du mouvement de révolution, ${ }^{8}$ il incite ses compatriotes à élargir leur lutte politique et nationale.

\section{L'IDÉE DE FÉDÉRATION IMPÉRIALE}

Dans l'esprit de cette "double lutte", Parent prie les Canadiens de rejeter toute velléité d'une possible adhésion de leur part à une fédération impériale, lorsque Pierre de Sales Laterrière se fait l'apôtre de l'idée de la représentation directe des colonies au Parlement de la métropole par deux députés membres de chacune des législatures provinciales, considérant que c'est le "seul moyen de rendre justice plénière et de contenter les habitants de ses diverses possessions qui, jusqu'à présent, ont généralement été considérés comme des gens conquis, et par [pour] cette raison n'ont pu se former ni s'attacher au caractère national ..." 9 L'avenir du Bas-Canada, majoritairement francophone, réside dans l'émancipation coloniale, indique Parent. Pour lui, le mal provient des principes appliqués au système colonial. Il expose sa solution:

... Il faudrait donc que le système d'exclusion et de monopole disparût c'est-à-dire que les colonies pussent elles-mêmes régler leur commerce, afin de donner à la législation cette fixeté [sic] et cette liberté sans lesquelles le commerce est comme l'enfant au maillot, sauf certains avantages particuliers pour le [sic] métropole, fondés sur l'intérêt des deux parties...

Il faudrait en second lieu que le système du patronage disparût aussi; c'est-à-dire que le régime gouvernemental fût tel, qu'une administration impopulaire, ou de minorité, fût chose impossible. Il faudrait que les colonies pussent participer aux précieux avantages que la réforme parlementaire va

${ }^{8}$ Louis Nourry, La pensée politique d'Etienne Parent, 1831-1852, thèse de Ph.D., [Histoire] (Université de Montréal, 1971). Comme le soutiendra Parent, le transfert du pouvoir exécutif aux mains des Canadiens aurait véritablement été une révolution dans le Bas-Canada.

9 Cité par Etienne Parent, Le Canadien, 10 août 1831. 
procurer au peuple anglais, qu'une classe privilégié [sic] ne pût à l'avenir exploiter à son profit des peuples entiers; il faudrait que nous eussions une administration Canadienne de sentimens; Canadienne d'intérêt, Canadienne même de préjugés, et nous disons ceci dans la persuasion où nous sommes, que si jamais ce pays cesse d'être Canadien, ce ne sera pas pour devenir Anglais ...10

Laterrière revient à la charge en anticipant dans un avenir rapproché l'inévitable assimilation des Canadiens. Il estime être "plus prudent... de marcher avec le temps... de conserver ce qui nous est utile, de former de bonnes institutions, et de mettre nos descendants en état d'être bien gouvernés et d'être heureux..." 11 Parent précise sa solution:

... Bien loin d'envoyer des représentans pour haranguer en parlement sur nos affaires, il faut désirer que l'Angleterre n'ait plus rien à décider sur notre politique intérieure...

l'état de ces dépendances, de celles au moins qui ont un vaste territoire, est celui d'une adolescence vigoureuse, qui court vers l'âge viril... l'existence coloniale n'est qu'un acheminement vers une existence plus noble et plus digne d'un grand peuple...

Pour nous, nous ne voyons qu'un seul plan efficace pour mettre fin à toutes les difficultés qui ont de tout temps existé entre les puissances Européennes et leurs possessions étrangères ou colonies, c'est l'émancipation progressive fondée sur la justice et l'intérêt réciproque... Entre l'époque de l'émancipation dont nous parlons et celle où une colonie peut prendre place parmi les nations et maintenir par elle-même son indépendance contre l'aggression [sic] étrangère, il s'écoulerait une période de temps pendant laquelle l'union entre les colonies et les mères-patries devrait encore subsister sur un principe d'intérêt réciproque: la colonie, par exemple, aurait à craindre les tentatives et les insultes des puissances étrangères, et pour la protection que lui donnerait la mère-patrie, elle accorderait à celle-ci des avantages commerciaux plus ou moins grands ...12

\section{L'IDÉE DE FÉDÉRATION AMÉRICAINE}

Le 12 octobre, Parent fait appel à l'intérêt de l'Angleterre pour demander de nouveau un protectorat canadien-français sous tutelle extérieure anglaise jusqu'à l'indépendance:

... Notre écrivain ne voit pour l'avenir de ce pays que deux [sic] alternatives, l'une d'une union éternelle avec l'Angleterre, et l'autre de devenir une province américaine... le peuple Canadien serait dès à présent flétri à jamais, s'il

10 Ibid., 13 août 1831.

11 Ibid., 5 octobre 1831.

12 Ibid., 8 octobre 1831. 


\begin{abstract}
n'osait penser à autre chose qu'à être tiré à la remorque d'aucune puissance au monde...

jamais assurément nous ne pouvons être une simple province américaine, mais nous pouvons bien, par la mauvaise politique de l'Angleterre, devenir, avant un grand nombre d'années, un des Etats de la confédération américaine. Une politique coloniale qui aurait pour but l'anglification sociale du BasCanada, serait le moyen le plus sûr de hâter cet événement, et la chose s'explique d'elle-même ...

... ce peuple qui a un grand fond de nationalité, qui veut conserver ses lois, ses coutumes et ses institutions, parce qu'elles l'ont rendu heureux, trouve son intérêt à demeurer uni à l'Angleterre, jusqu'à ce qu'il soit en état de maintenir son indépendance par lui-même, surtout contre l'ambition de ses voisins qui sont ou seront bientôt... des rivaux redoutables pour l'empire dont il fait partie. Ce peuple qui n'est encore que dans l'adolescence redoute une union avec la nation voisine ... Ce n'est pas qu'il haïsse de cœur ou méprise ses voisins, au contraire il les admire, mais semblable au Corrège voyant un tableau de Raphaël, il se dit "Et moi aussi je suis peintre !"...

rassurons-nous, la mère-patrie sentira tôt ou tard que de notre nationalité dépend en partie l'allégeance de cette colonie...13
\end{abstract}

Parent refuse donc toute mise en minorité dans une fédération impériale ou dans la fédération américaine. Les Canadiens doivent plutôt exiger de l'Angleterre une "quasi-indépendance" 14 avec un status voisin de celui d'un protectorat sous l'autorité nominale de la Couronne britannique. Toutefois, à défaut de cette obtention et face à une "politique coloniale qui aurait pour but l'anglification sociale du Bas-Canada", ils seraient contraints de se joindre à la fédération américaine où ils pourraient jouir de l'autonomie intérieure. Aussi, les Canadiens, devant aspirer à l'indépendance, ne peuvent conséquemment accepter "une union éternelle avec l'Angleterre" dans une fédération impériale. Cependant, croit-il naïvement, ils pourront sortir de la fédération américaine s'ils n'ont pas encore été assimilés, lorsque le moment de leur indépendance sera venu. Parent écrira en 1835 :

... il résulte, que d'ici à un certain nombre d'années, jusqu'à ce qu'il puisse former une puissance indépendante si la providence lui réserve ce sort dans l'avenir, le Canada devra faire partie de celui des deux empires, le Britannique ou l'Américain, qui lui assurera l'existence politique la plus conforme aux goûts et aux besoins de la population du pays, puisque de sa coopération ou de son opposition dépend le règne de la puissance souveraine...15

13 Ibid., 12 octobre 1831.

14 Ibid., 29 mars 1833.

15Ibid., 18 septembre 1835. 


\title{
L'IDÉE DE FÉDÉRATION CANADIENNE
}

En 1833, lors d'une discussion avec La Gazette de Québec qui s'enquérait des institutions américaines que le parti patriote souhaitait implanter dans le Bas-Canada, Parent précisait le moment de l'accession à l'indépendance pour les Canadiens:

\begin{abstract}
... Le Canada est une colonie, qui est destinée à former un vaste empire, et pour lequel conséquemment l'état colonial n'est qu'un état de transition, et il n'est pas probable, il est même impossible que dans un demi-siècle il soit encore colonie Européenne, et si l'Angleterre veut reculer cet événement, ce n'est que par une politique libérale qu'elle peut le faire. Il est donc de son intérêt de se rendre aux vœux du peuple lorsqu'il demandera quelque amélioration dans sa constitution, qui étant faite pour un pays destiné à l'indépendance, doit naturellement subir des modifications à mesure que le peuple approche du but...16
\end{abstract}

La Gazette de Québec rétorque aussitôt et lui "conseille de partager... [sa] loyauté en cinquante parties, de crainte d'en manquer avant la fin du demi-siècle". 17 Elle déclare avoir "toujours compris que la loyauté ou, pour mieux dire, la fidélité que les sujets doivent au Souverain, est un principe de morale, une obligation qui ne cesse point, que par l'impossibilité où l'on se trouve de s'en acquitter..."18 Parent lui oppose la morale politique proclamée par le "Génie de l'Amérique" jusqu'au $45^{\mathrm{e}}$ degré nord:

... Il y a donc une autre loi, une autre morale qui est la base de l'union de ces colonies avec la métropole, et nous n'en voyons pas d'autre que celle qui se trouve renfermée dans ces deux mots "Protection et Suprématie", et notre morale, c'est que celle-ci doit cesser aussitôt que l'autre n'est plus nécessaire. Toute scission violente d'un pays d'avec un autre est venue de ce qu'une partie n'a pas voulu reconnaître ce droit naturel des peuples ...19

Puis, Parent réfute l'objection selon laquelle dans cinquante ans les Canadiens ne seront pas assez nombreux pour être en état et en droit de demander l'indépendance:

... La nature et leur intérêt réciproque enseigneront aux pays qui bordent le Saint-Laurent de former une espèce de confédération, soit à l'instar de celle des Etats-Unis, soit sur d'autres principes plus conformes à leur: situation et à leurs

16 Ibid., 10 avril 1833.

17 Ibid., 12 avril 1833.

18 La Gazette de Québec, 13 avril 1833, citée par Etienne Parent, ibid., 15 avril 1833.

19 Ibid., 15 avril 1833. 
mœurs, et c'est ce qui arrivera probablement aussitôt que le temps de leur indépendance sera venu. Eh bien, cette étendue de pays où l'on compte aujourd'hui deux millions d'hommes environ, ne contenaient [sic] en 63 qu'environ 75 à 80,000 âmes, et en supposant que la population augmente dans la même proportion qu'elle l'a fait depuis 63 , il y aura dans 70 ans une population de vingt millions. Mais si l'émigration continue, pendant cet espace de temps, sur le pied sur lequel elle va depuis quelques années, on peut porter en toute sûreté à environ vingt-cinq millions d'âmes la population des colonies de l'Amérique Septentrionale dans 70 ans d'ici ...20

Lorsque William Lyon Mackenzie propose, dans un volume publié à Londres sous le titre Mackenzie's Sketches of Canada and The United States, qu'il se tienne à Québec "une Conférence par des délégués du peuple de chaque colonie, dont les attributions seraient de délibérer sur toutes les affaires qui concernent plusieurs colonies ou toutes les colonies ensembles [sic], comme les communications intérieures d'une colonie à l'autre, les poste, [sic], l'émigration, les terres, \&c.". ${ }^{21}$ Parent accueille favorablement cette offre:

Ce projet est si vaste, si fécond en conséquences, qu'il est difficile d'y concourir au premier abord; mais il mérite assurément d'attirer toute l'attention de nos hommes d'état. Nous dirons cependant que notre première impression lui a été favorable, d'autant plus que nous parlions il $y$ a quelque temps que l'intérêt commun des colonies les ferait concourir un jour, lorsque la protection immédiate de la Mère-Patrie leur serait devenue moins nécessaire, à former entre elles une espèce de confédération, pour être en état de résister aux convoitises de voisins trop puissants pour chaque colonie isolée. Nous reviendrons sur le sujet.22

Par crainte des Etats-Unis, Parent accepterait donc que ses compatriotes qui, selon lui, doivent repousser l'idée de fédération impériale et celle de fédération américaine en conservant comme objectif ultime l'indépendance, participent, au moment où elle leur sera accessible, à la formation d'une fédération canadienne. Il consentirait à ce que les Canadiens partagent au niveau du gouvernement fédéral, avec une majorité britannique gros-

20 Ibid., 15 avril 1833.

21 Ibid., 21 août 1833.

22 Ibid., 21 août 1833. La semaine suivante, le 30 août, il rapporte cette réaction de $L a$ Minerve à la proposition de Mackenzie: "Et qui empêcherait de concevoir l'dée [sic] d'une fédération modelée sur celles [sic] des puissantes républiques qui nous avoisinent, et au moyen de laquelle le peuple de chaque province, réglant librement ses affaires particulières, ferait cause commune avec celui de toutes les autres pour tout ce qui aurait rapport aux intérêts généraux..." 
sie par l'immigration, les pouvoirs se rapportant à la concession des terres, à l'établissement de nouveaux. habitants et "plusieurs autres" ${ }^{23}$, tous aussi essentiels à la préservation et à l'épanouissement de la "nation canadienne".

Avant qu'il ne "revienne sur le sujet", les "92 Résolutions" seront dévoilées devant les membres de la Chambre d'assemblée du Bas-Canada, le 15 février 1834. Parent se fait l'acclamateur, l'interprète, le stratège, l'apôtre et le défenseur de ce "manifeste foudroyant" 24 qui réclame la formation d'une république canadienne-française virtuelle. Ainsi, lors de la campagne électorale de l'automne, il priera chacun de ne voter que pour le candidat qui, "dans les circonstances actuelles ...., pense, sur les grandes questions du jour, comme la dernière Chambre d'Assemblée et la très grande majorité du peuple". ${ }^{25}$ Il appuiera la nouvelle majorité canadienne accrue, quand elle vient rapidement en conflit avec Lord Aylmer et le Conseil législatif, au cours de la session de 1835.

$\mathrm{Au}$ début de l'été, Londres nomme un nouveau gouverneur, Lord Gosford, en lui adjoignant Sir George Gipps et Sir Charles Grey pour mener l'enquête dont il est chargé. Le 27 octobre 1835, dans son discours d'ouverture des Chambres, Lord Gosford donne cet avis aux Canadiens:

$\ldots$ Ne craignez pas que l'on ait dessein de troubler la forme de société sous laquelle vous avez si long-temps joui du contentement et de la prospérité... l'Angleterre ne peut qu'admirer les arrangemens sociaux par lesquels on est parvenu $\grave{a}$ faire d'un petit nombre de colons industrieux, une race d'agriculteurs bons, religieux et heureux...26

23 Dans une lettre qu'il adressait à John Neilson, datée à York, le 7 décembre 1829 , Mackenzie spécifiait quelques autres pouvoirs dont il désirait voir l'Etat fédéral investi: “... I am desirous to see us more our own masters in regard to our local concerns, and think that while the local legislatures are continued in each province, a federal system might be now set on foot between the colonies by which their connection with England might be at once rendered more secure, permanent, and beneficial to both countries while their slavish dependence on her ministers would be done away. The currency, the post-office, the bankrupt laws, the poor laws, the naturalization laws, post-roads, and many other things now unattended to or ill-done, would be perhaps properly placed, I think, in the hands of a federative union..." Margareth Fairley, The Sielected Writings of William Lyon Mackenzie 1824-18:37 (Toronto, 1969), 291.

24 Etienne Parent, Le Canadien, 12 janvier 1835.

25 Ibid., 10 octobre 1834.

26 Lord Gosford, Journaux de la Chambre d'assemblée dw BasCanada (session 1835-6), 45: 14 . 
Rassuré sur le maintien des caractères nationaux des Canadiens, Parent préconise aussitôt un rapprochement entre la Chambre d'assemblée et le gouverneur. Il souhaite qu'on feutre les revendications d'ordre politique durant "une année" 27 et assure à l'Angleterre "une possession séculaire des bords du St-Laurent". ${ }^{28}$ Les chefs patriotes ne veulent rien entendre. Ils l'accuseront "de faire du modérantisme", ${ }^{29}$ de défendre "le chef du gouvernement $\grave{a}$ tort et $\grave{a}$ travers" ${ }^{30}$ et "de faire l'esclave, le chien-couchant aux genoux des Ministres". ${ }^{31}$

Au début d'avril 1837, on prend connaissance de la réponse métropolitaine aux "92 Résolutions". Lord John Russell refuse l'octroi du "Responsible Government" et menace d'autoriser le gouverneur à puiser dans les coffres publics pour payer les arrérages du gouvernement civil. Parent invite alors ses compatriotes à "réparer les avaries de la tempête". ${ }^{32}$ Il regrette qu'on ait "voulu pousser jusqu'à Moscou" ${ }^{33}$. Les chefs patriotes le vouent aux gémonies et tiennent des assemblées publiques au cours desquelles ils démontrent leur incapacité de jauger la conjoncture. Après la session avortée de l'été, lors d'un nouvel appel à la prudence et à la temporisation, Parent entrevoit la possibilité de l'entrée des Canadiens dans la fédération américaine ou dans une fédération canadienne:
... Bien assurément, quel que soit l'avenir que nous réserve la providence, que nous devions un jour prendre place au rang des nations, ou que nous devions faire partie de la grande fédération qui nous avoisine, ou en former une nouvelle avec les peuples du fleuve et du golfe; dans tous les cas il est de l'intérêt de la nationalité française en Amérique, que notre séparation de l'Angleterre ne soit pas précipitée, et tous les efforts des vrais patriotes Canadiens devraient se tourner vers ce but, au lieu d'être dirigés en sens contraire...34

Le conflit armé donne à la métropole l'occasion de suspendre la Constitution du Bas-Canada. Lord Durham vient mener une enquête sur l'ensemble du "British North America". Après avoir débarqué à Québec le 29 mai 1838 et séjourné à Montréal, il se rend, en juillet, visiter le Haut-Canada. Lorsqu'il apprend

27 Etienne Parent, Le Canadien, 16 novembre 1836.

28 Ibid., 11 novembre 1836.

29 Ibid., 7 mars 1836.

${ }^{30}$ La Minerve, 15 août 1836, citée par E. Parent, ibid., 19 août 1836.

31 Le Vindicator, 10 mars 1837, cité par E. Parent, ibid., 15 mars 1837. 32 Etienne Parent, Le Canadien, 19 avril 1837.

33 Ibid., 17 juillet 1837.

34 Ibid., 1er septembre 1837. 
que Durham, de passage à Cornwall, aurait avoué son désir d'unir toutes les colonies du "British North America", Parent spécule:

...Son Excellence est en faveur du plan de confédération dont il est question depuis quelque temps. L'adoption de ce plan jetterait les fondements d'un nouvel empire sur ce continent, et fixerait l'incertitude où sont ces colonies sur leur avenir. La perspective que cette mesure leur ouvrirait serait assez flatteuse pour les empêcher de se laisser entraîner à d'autres espérances moins en harmonie avec les intérêts de l'Empire. 35

La Montreal Gazette se dit bientôt autorisée à déclarer que "Son Excellence le Gouverneur Général n'a fait aucune allusion, dans sa réponse à l'adresse de Cornwall, à l'union des provinces, ni à aucune mesure législative quelconque..." 36 Cependant, il appert par la dépêche "secrète" de Durham à Glenelg en date du 9 août 1838, que le célèbre enquêteur caresse effectivement le plan d'une union législative ou fédérale des colonies du "British North America".

Dans cette dépêche, lord Durham dénonce la politique impériale depuis 1763 qui a encouragé le fait français dans la grande "Province of Quebec" et, après 1791, dans le Bas-Canada. Il plaide la mise en tutelle des Canadiens français: il faut soustraire toute législation se rapportant aux grands intérêts britanniques de l'emprise d'une majorité canadienne-française sans violer les droits mineurs des sujets conquis, tels leurs lois, leur langue et leur religion; il faut instituer une union fédérale ou législative des colonies du "British North America" afin de pouvoir instaurer les principes les plus sûrs d'un gouvernement constitutionnel à l'image de celui de Londres ${ }^{37}$.

Après les commentaires de Parent sur la prétendue déclaration de Durham à Cornwall, La Gazette de Québec présume que "le Canadien ne serait pas éloigné d'accepter l'union" 38. En avouant sa préférence pour l'union fédérale, Parent distingue alors entre les conséquences pour les Canadiens de l'union fédérale de toutes les colonies et celles de l'union législative des Canadas: "avec la première nous conservons notre législature locale pour notre législation locale, et, avec l'autre, nous la perdons,

35 Ibid., 16 juillet 1838.

36 The Montreal Gazette, citée dans Le Canadien, 27 juillet 1838. 350-362.

37 Rapport des Archives Publiques pour l'année 1923 (Ottawa, 1926),

38 La Gazette de Québec, citée par E. Parent, 18 juillet 1838. 
nos affaires locales tombent sous la jurisdiction [sic] conjointe d'une autre province" 39 . Effectivement, les Canadiens obtiendraient de nouveau un Etat séparé, mais ils n'y détiendraient que des pouvoirs mineurs qui ne leur permettraient qu'une vie nationale étiolée. Parent termine sa réplique en se disculpant de désirer le démembrement prochain de l'Empire britannique:

\begin{abstract}
... il nous semble que nous nous montrons plus ami de l'Empire Britannique, en accueillant un plan qui créerait sur ce continent une espèce de protectorat pour l'Angleterre, qu'en préférant laisser ces provinces au hasard d'être un jour agglomérées à une puissance rivale de l'Angleterre. Il n'est pas un homme d'état chez la Mère-Patrie qui rêve à une souverainetá permanente pour elle sur ces colonies; il y en a beaucoup qui croient à la possibilité d'une séparation à l'amiable, dans un avenir plus ou moins éloigné. Or, cette époque arrivée, il importe à la Grande-Bretagne que ces colonies aient été habituées à régler entre elles leurs affaires inter-coloniales, à se gouverner, pour ainsi dire elles-mêmes, car le défaut de concert entre elles en pareille occurrence les livrerait à la puissance voisine. Il est même de l'intérêt du monde entier que la même puissance ne possède pas le Mississipi, l'Hudson et le St. Laurent. Nous nous trompons fort, ou c'est là la pensée qui est au fond du plan de la confédération de ces colonies, et la Gazette, dans son puéril dévouement, se montre peut-être plus Britannique que ne le permet l'intérêt de la Grande-Bretagne elle-même.40
\end{abstract}

La Gazette de Québec oppose maintenant cette déclaration de Parent à celle de Durham dans sa réponse à une adresse présentée par les notables de Toronto: “... Ces provinces sont quelques-uns des plus précieux ornements de la Couronne de la Grande-Bretagne; leur éternelle connexion avec cette Couronne devrait être l'objet de chaque homme d'état Britannique, qui met du prix à la sûreté et à la prospérité de l'Empire..." ${ }^{41}$ Les idées de fédération et de "connexion éternelle", voudra expliquer Parent, peuvent se concilier: il ne faut pas prêter à Lord Durham "l'idée absurde que les colonies de ce continent peuvent demeurer en connexion éternelle, dans le sens absolu du mot, avec la Grande-Bretagne, dans l'état de dépendance coloniale et sans qu'il s'opère des changements notables dans leurs rapports réciproques" ${ }^{42}$. Il interprète bien la pensée du précurseur de l'idée de "Commonwealth": fédérées, les colonies du "British North America" seront plus fortes; plus fortes, elles seront plus égales à l'Angleterre; et, plus égales à l'Angleterre, elles pour-

39 Etienne Parent, Le Canadien, 18 juillet 1838.

40 Ibid., 18 juillet 1838.

41 Lord Durham, ibid., 27 juillet 1838.

42 Ibid., 27 juillet 1838. 
ront entretenir avec cette puissance des relations diverses plus étroites, pour une période de temps indéfinie.

Il vaudrait mieux, suggère-t-il, rétablir dans le Bas-Canada un régime représentatif où la majorité gouvernerait de façon libérale, "mais si l'on introduit quelque modification dans le système colonial, nous préférerons celui qui affectera le moins l'existence sociale de cette classe [de colons qui nous touche de plus près], et de tous les plans dont on a parlé, celui de la confédération nous a paru offrir le moins de danger".43

Lorsque La Gazette de Québec relance le débat, Parent étaie son interprétation des mots "connexion éternelle" à l'aide d'une déclaration de Lord Howick, ${ }^{44}$ ministre de la guerre, lors des discussions au Parlement impérial sur l'utilité ou la nécessité de suspendre la Constitution dans le Bas-Canada. "Vu l'espace presque illimité qui est laissé à l'augmentation de la population en Canada, déclare Lord Howick, un temps doit nécessairement arriver à la fin où la grandeur relative des deux nations serait entièrement changée, et les rapports de mère-patrie à colonie entre elles seraient absurdes en pratique." 45 Il croyait aussi, rapporte Le Canadien, que "l'union entre la métropole et le Canada subsisteraient [sic] un nombre d'années presque infini" "46. Parent approuve donc cette prédiction de Lord Howick et répète que les Canadiens tiennent à demeurer séparés, mais, s'il leur faut s'unir, ils préféreront alors la fédération:

\#. il n'est pas de l'intérêt de nos compatriotes de hâter l'époque de la séparation; situés comme ils sont, leur intérêt au contraire, est de la retarder; mais cela ne doit pas les empêcher, afin de n'être pas pris au dépourvu, d'examiner et de calculer la marche des choses, qui souvent renverse les plus belles spéculations, comme les vœux less plus doux...47

On apprend bientôt que les représentants de chacune des colonies du Golfe, mandés par Durham à Québec, auraient donné leur adhésion à un plan de fédération de l'ensemble des colonies du "British North America". Après avoïr souhaité qu'on revienne au régime de 1791 dans le Bas-Canada, en laissant présager que la majorité canadienne-française gouvernerait de façon

43 Ibid., 27 juillet 1838.

44 Beau-frère de Durham, il accordera, sous le nom de Lord Grey, la responsabilité ministérielle aux colonies du "British North America" le 3 novembre 1846. W. P. M. Kennedv, Documents of the Canadian Constitution 1759-1915 (Toronto, 1918), 570-573.

45 Lord Howick, ibid., 1er août 1838.

46 Ibid., 1 er août 1838.

47 Ibid., 1er août 1838. 
libérale, et après avoir considéré que l'union fédérale était la moins dangereuse des formules d'union pour les Canadiens, $\mathrm{Pa}-$ rent adhère maintenant davantage à l'idée de fédération canadienne. Toutefois, celui qui voit dans la conquête de 1760 "une adoption honorable et avantageuse pour nous", ${ }^{48}$ espère que "Mother England" protégera "nos institutions, notre langue et nos lois" dans les futurs cadres fédéraux:

Nous avons déjà dit qu'en principe nous n'aurions aucune objection à un pareil plan, qui nous paraîtrait offrir de grands avantages aux Colonies, d'abord pour le règlement de leurs intérêts communs entre elles, et en second lieu pour le règlement de leurs dîfficultés avec leur Administration locales [sic] ou avec les autorités Impériales, sur les sujets qui intéresseraient toutes les colonies. Il y aurait alors concert et unité d'action entre toutes, et cela ne manquerait pas de donner plus de force et de poids à leurs représentations, qu'elles n'en ont aujourd'hui, qu'elles agissent séparément, isolément, et en différents temps. Voilà pour le présent. Quant à l'avenir, une pareille mesure jetterait sur les bords du St. Laurent, les germes d'une puissance capable sous peu de temps de se maintenir et de se protéger au besoin contre toute agression du dehors, faculté que ces colonies ne peuvent se flatter d'avoir séparément d'ici à une bien longue suite d'années. Et c'est une preuve des vues libérales [c'est-à-dire l'intention de recommander l'octroi d'un gouvernement libéral] de Lord Durham, que la conception ou l'adoption de ce plan de sa part, car la puissance coloniale qu'il décuplerait ainsi pourrait s'exercer contre la métropole elle-même, si celle-ci refusait de leur rendre justice, et on n'augmente jamais la force de ceux que l'on veut opprimer.

Nous ne voyons dans l'arrangement aucun danger pour les intérêts et droits particuliers à la défiance [sic] desquels nous nous sommes plus spécialement voué, car ces intérêts et ces droit [sic] resteraient sous la compétence de la Législature locale, qui serait tout-à-fait indépendante de la Législature fédérale, et de plus, tout comme à présent, sous la sauvegarde de l'honneur et de la foi jurée de la Métropole ...49

Le 19 septembre, Durham apprend par les journaux américains que son ordonnance d'amnistie du 28 juin a été désavouée par le cabinet Melbourne. Le 25, il écrit sa lettre de démission à Glenelg, secrétaire d'Etat aux colonies. Les constitutionnalistes de Montréal convoquent une assemblée de leurs troupes pour le 1er octobre et ceux de Québec, pour le 3 octobre, afin de préparer des adresses d'adieu. A l'assemblée de Montréal, Durham délègue Adam Thom, le fougueux rédacteur du Montreal Herald de

48 Ibid., 7 mai 1831.

49 Ibid., 24 septembre 1838. 
1835 à 1838," ${ }^{\text {0 }}$ en lui confiant la mission d'amener les "Montrealers" à accepter son plan de fédération des colonies du "British North America".

Thom commence par dévoiler l'intention de Durham de prier le Parlement impérial de provoquer l'assimilation des Canadiens français. Puis, il expose que, par suite d'une alliance majoritaire entre les Canadiens français et les réformistes hautscanadiens, l'union législative des Canadas pourrait être un échec irréparable. Pour éviter cet échec, il voudrait ajouter aux partisans de cette union des Canadas "une nouvelle somme de sang et de sentiments britanniques" que l'on pourrait trouver en "masses solides" à la Nouvelle-Ecosse et au Nouveau-Brunswick. Il incite ses auditeurs à ne pas s'effrayer de l'épithète fédérale et les informe enfin que Lord Durham est désireux de recevoir leurs suggestions. ${ }^{51}$

Les "Montrealers" exigent une fois de plus l'union législative des Canadas car ils craignent d'être de nouveau brimés, par une majorité canadienne-française dans le Bas-Canada, même avec l'assurance d'un Etat central qui détiendrait tous les grands pouvoirs. Après avoir accepté une possible union fédérale "basée sur des principes de justice et d'égalité envers toutes les parties intéressées",52 Parent s'indigne à la lecture du discours de Thom. Il accuse Durham de vouloir pervertir le régime fédéral, solution libérale au problème posé par l'affirmation nationaliste canadienne-française:

... lorsqu'il est transpiré dans le public que lord Durham était en faveur d'une confédération des cinq colonies, tout le monde a pensé qu'il n'entrait aucune vue ou fin de parti dans cette mesure; que la position et les intérêts particuliers des partis, dans chacune des colonies confédérées, ne souffriraient aucune atteinte de la législature fédérale, et que sous ce rapport les choses resteraient in Statu quo; mais voici que M. Thom... nous apprend que le grand objet de cette mesure serait d'assurer la prépondérance aux soi-disant Loyaux, vue mesquine, vue oppressive, vue indigne en tout d'un homme d'Etat libéral ...53

La vive expression du sursaut de nationalisme auquel il est en proie au cours des semaines suivantes, au moment même où

50 Pour une analyse de ses articles les plus virulents, voir André Lefebvre, Les "Montrealers" et la crise politique du Bas-Canada (juillet 18.35-mars 1836), mémoire prédoctoral (Université de Montréal, 1960), 190 pages.

51 Adam Thom, cité par Etienne Parent, Le Canadien, 8 octobre 1838.

52 Etienne Parent, Le Canadien, 24 septembre 1838.

53 Ibid., 8 octobre 1838. 
un second soulèvement se produit dans la région sud de Montréal, le conduit à la prison de Québec le 26 décembre 1838. Lorsqu'il est libéré quatre mois plus tard, il présente une traduction du Rapport Durham dans Le Canadien, il préconise l'assimilation volontaire à ses compatriotes et les incite à s'unir aux réformistes du Haut-Canada dans le but de conquérir le "Responsible Government".

A cette époque, Francis Hincks, rédacteur de l'Examiner de Toronto, plaide auprès de LaFontaine la nécessité pour les Canadiens français de se joindre, dans cette lutte, aux réformistes du Haut-Canada, "as Canadians". ${ }^{54}$ Lorsque LaFontaine indiquera sa préférence pour une union fédérale, Hincks s'efforcera de le circonvenir en affirmant que l'idée de Durham de former des conseils de district renferme celle de fédération. Que seraient ces conseils de district sinon une législature sans Conseil législatif élisant son propre président, demandera-t-il ? 55

Dans Le Canadien, Parent commentera favorablement quelques éditoriaux de l'Examiner à saveur pancanadianiste. ${ }^{56}$ Toutefois, après la lecture des cinq résolutions en faveur de l'union législative des Canadas, présentées au gouverneur Poulett Thomson le 14 décembre par le Conseil législatif du Haut-Canada, puis des quatre résolutions présentées le 23 par la Chambre d'assemblée, et, enfin, de l'adresse à la Reine, adoptée le 13 janvier 1840, et des débats qui furent soulevés à la Législature de cette province, Parent s'élève contre l'animosité déployée à l'endroit des Canadiens français et contre certaines clauses qu'on veut voir inscrites dans la nouvelle Constitution. Lors d'une réunion tenue le 17 janvier 1840 chez Glackmeyer, à Québec, il prête son concours à Thomas-Cushing Aylwin, François-Xavier Garneau, Ronald McDonald et John Neilson dans la rédaction de treize résolutions qui seront adoptées à une assemblée publique tenue le 24, à l'Ecole des Glacis. Dans la dernière résolution, on demande le maintien de la Constitution de 1791.57

Une fois votées, ces résolutions sont reproduites dans une requête ou pétition adressée à la Reine et aux deux Chambres du Parlement impérial. Au mois d'avril, Vital Tétu, marchand de Québec et ex-député de Montmorency, est chargé de la porter à Londres. Il n'y obtiendra aucun succès. Après avoir été adopté

54 Voir particulièrement les lettres de Hincks à LaFontaine du 12 avril et du 9 septembre 1839 dans la collection LaFontaine.

55 Hincks à LaFontaine, 9 octobre 1839, ibid.

56 Le Canadien, 17 juillet, 7 août et 25 septembre 1839.

57 Le Canadien, 27 janvier 1840. 
à la Chambre des Lords le 20 juillet et à la Chambre des Communes le 22, le Bill d'Union de Lord John Russell devient l'Acte d'Union, $3 \& 4$ Victoria, ch. 35.

Aussitôt que les soixante-deux articles sont connus dans la colonie, Parent émettra l'opinion de choisir pour représentants à l'Assemblée du Canada-Uni des hommes qui seraient disposés à demander la révocation ou l'amendement de l'Acte d'Union. ${ }^{58}$ L'idée fait tache d'huile. Le 14 octobre, se tient à l'Ecole des Glacis, une assemblée publique où on adopte un projet de "Lettre des électeurs de Québec qui désapprouvent l'Acte pour réunir les deux provinces, à tous les électeurs de la province". Parent demande fortement à ses lecteurs d'appuyer ce "manifeste du parti libéral ou réformiste du Bas-Canada". ${ }^{59}$ Puis, quelques semaines plus tard, il profite de ce que les habitants du CapBreton exigent la révocation de leur union avec la NouvelleEcosse pour solliciter la formation d'une fédération canadienne:

Pendant qu'O'Connell fait tressaillir l'Irlande de son cri patriotique de 'Rappel de l'Union', voilà que le même cri, le même vou s'élève au sein de l'Ile du Cap-Breton ... Pourtant on aurait pu penser que... tout ce qui peut constituer une similitude nationale parfaite auraient [sic] assuré la cimentation de l'union du Cap-Breton avec la Nouvelle-Ecosse... Le fait est qu'au lieu d'unir des provinces et de concentrer ainsi le pouvoir, l'intérêt de ces coloniess comme celui de la métropole demanderait plutôt des subdivisions, la formation de nouvelles provinces séparées à mesure que les établissements et leurs besoins s'augmentent...

Il serait à souhaiter que les hommes d'Etat de la Métropole, au lieu de consulter leurs propres idées, fruit d'un état de choses différent de celui d'Amérique, ou de se rendre aux inspirations d'hommes de parti violents, avides et ambitieux dans les colonies, consultassent davantage les procédés des hommes d'état des Etats-Unis. Des hommes et un système qui ont fait les Etats-Unis ce qu'ils sont ne sont pas à mépriser, surtout dans les parties du système en question qui a complètement réussi. En multipliant les centres de pouvoir et de gouvernement ils ont créé un grand nombre d'états populeux, florissants et unis entre eux. Il $\mathrm{y}$ a cent à parier contre un qu'en suivant un système opposé, l'Angleterre nous prépare des conséquences aussi tout opposées. ${ }^{60}$

Parent fausse ici cette question. Lord Durham, cet "homme d'Etat de la Métropole", avoue, dans son Rapport, qu'il favorisait d'abord une fédération des provinces du "British North

58 Dans sa célèbre "Lettre aux électeurs du comté de Terrebonne" du 25 août 1840, LaFontaine ne demande que l'amendement de l'Acte d'Union.

59 Etienne Parent, Le Canadien, 16 octobre 1840.

60 Ibid., 7 décembre 1840. 
America", mais qu'il a dû recommander une union législative des Canadas pour deux raisons principales: la crainte d'une obstruction systématique de la part des Canadiens français à l'endroit de l'Etat fédéral et le refus des "Montrealers" de l'union fédérale. De plus, Durham voulait que Londres accorde la régie intérieure, sauf celle des terres publiques, à une majorité réformiste et non à des "hommes de parti violents, avides et ambitieux".

Deux jours plus tard, Parent informe ses lecteurs de la demande de "la Nouvelle Galles du Sud appelée Australie Heureuse... à être séparée de la partie Nord... [en alléguant] l'éloignement des lieux, la difficulté des communications, et la diversité des intérêts entre les deux sections". ${ }^{61}$ Il professe de nouveau sa foi dans la supériorité de l'union fédérale sur l'union législative:
... Cette demande constitue une nouvelle preuve de l'incon- venance d'unir sous un seul gouvernement de grandes étendues de pays nouveau, où la difficulté des communications, et la multiplicité des besoins demandent au contraire des jurisdic- tions [sic] gouvernementales aussi restreintes que possible. Si pour de hautes raisons de politiques générales, il devenait nécessaire de réunir plusieurs de ces pays sous une seule jurisdiction, ce ne devrait être que pour des fins limitées et générales, et dans ce cas ce serait le système fédératif qu'il faudrait adopter...
Rappel d'union en Irlande, rappel d'union au Cap-Breton, rappel d'union à l'Australie Heureuse, et désaveu de l'union dans le Bas-Canada, tout cela n'est pas un lit de rose pour M. Thomson et Lord John Russell: les Barings [sic] seuls retireront quelque chose de l'expérience. ${ }^{62}$

$\mathrm{Au}$ cours de la première session de la législature unie, les inspirateurs du "désaveu de l'union dans le Bas-Canada" ne font qu'enregistrer leur protêt contre certaines clauses de l'Acte d'Union. Mais, Parent profitera de la publication des résultats du recensement effectué aux Etats-Unis en 1841 - la population s'y élève à 17,069,453 âmes - pour ressasser la nécessité de la formation d'une fédération canadienne:

... nos gouvernants ... doivent voir la nécessité qu'il y a pour eux d'élever dans l'affection du peuple de ces colonies un rempart solide contre la pression de la masse énorme de population qui pèsera bientôt sur nos frontières. La création d'une nationalité assise sur de larges bases et nourrie de grandes espérances serait, à notre avis, le seul moyen d'empêcher ces colonies d'être entraînées dans le tourbillon

61 Ibid., 9 décembre 1840.

62 Ibid., 9 décembre 1840. 
de l'Union Américaine. Lord Durham en a eu l'idée d'abord. Une Confédération composée des deux Canadas et des Provinces du Golfe, avec une législature locale pour chaque Province sur le modèle de l'Union Américaine, est un plan qu'on ne devrait pas perdre de vue. L'union des Canadas nous a toujours paru être un acheminement à l'absorbtion [sic] de ces Province [sic] par les Etats-Unis; rmais il en serait tout autrement d'une confédération de toutes ces Provinces, qui seraient pour [sic] là mises dans la voie d'un avenir grand et certain, et travailleraient en conséquence à l'accomplissement de leur mission ou destinée, celle de fonder un empire ou état puissant sur les eaux du fleuve et du golfe St. Laurent... Quand on ... voit [nos gouvernants] à chaque pas tomber de Caribde [sic] à Scylla; pour éviter un danger minime et passager en préparer un permanent et fatal; travailler à l'anglification du Bas-Canada, c'est-à-dire à son assimilation avec les peuples des Etats-Unis, pour éviter une union du Canada avec les Etats... le plus sage parti est de fermer la bouche et de laisser faire ...63

Parent se fourvoie ici: Durham voulait l'assimilation des Canadiens français, leur mise en tutelle et la formation d'une nation "British-American", soutenue par l'Angleterre, dont le nombre s'accroîtrait rapidement grâce à une forte immigration et qui serait capable de résister à l'attraction américaine. Il n'y a pas dans ce but légitime, une bévue, comme Parent le laisse entendre. De plus, si l'Acte d'Union, à l'encontre des valeurs démocratiques, donne une représentation égale à chacune des deux provinces, il n'établit pas un "ordre de choses absurde". ${ }^{64}$ Il soude législativement et exécutivement deux provinces britanniques; il met entre les mains d'une majorité britannique le développement d'une même région éconornique. Enfin, par l'Acte d'Union qui épouse bien la réalité, une majorité britannique contrôle maintenant le développement d'un territoire peu peuplé que seule, avec l'aide de sa métropole naturelle, elle peut mener à bonne fin. C'est l'application de l'une des devises de Parent, "l'intérêt du grand nombre". ${ }^{65}$ Ce dernier soutient enfin:

Séparés, chacun avec sa législature locale, les Canadas auraient eu chacun sa puissance naturelle. Unis, malgré une des parties, ils n'auront pas ensemble la puissance que chacun aurait eue séparément. Il y avait, il y a un seul principe de force par voie d'agrégation pour ces colonies, c'est le principe fédératif, avec législation locale indépendante pour chaque province ou section. ${ }^{66}$

Ce souhait sera bientôt partiellement réalisé. En effet, lorsque LaFontaine devient procureur général du Canada-Est, le

63 Ibid., 16 février 1842.

64 Ibid., 16 février 1842.

65 Ibid., 7 juin 1833.

66 Ibid., 16 février 1842. 
16 septembre 1842, l'administration de cette section du CanadaUni relève maintenant de lui. Il n'est entré au Conseil exécutif de Bagot qu'à cette condition expresse. ${ }^{67}$ Le caractère fédéral imprimé à l'Union législative de 1840 émerge nettement. ${ }^{68}$ Parent notera: “... C'est la première fois que le peuple Canadien se trouve dans la position où il est aujourd'hui ... Cette nouvelle position, ce nouveau rôle exigent de sa part, plus de prudence, plus de modération que jamais, surtout une grande confiance dans ses chefs ..." 69

Augustin-Norbert Morin devient commissaire des terres de la Couronne avec un siège au Conseil exécutif. A l'aide du patronage, ${ }^{70}$ les chefs canadiens-français pourront endormir la petite bourgeoisie tandis que le clergé de Montréal poursuivra l'implantation de "la réaction catholique" ${ }^{71}$ qu'il vient d'amorcer dans le peuple. Etienne Parent accède au poste de greffier du Conseil exécutif, remet le mandat de député de la circonscription électorale du Sagu $\in$ nay qu'il détenait depuis le mois de mars 1841 et quitte la direction du Canadien le 21 octobre 1842. Entre 1844 et 1852 , il prononce dix conférences dont cinq devant les membres de l'Institut Canadien de Montréal.

Celui qui s'était avéré le grand théoricien du nationalisme politique canadien-français par ses raisonnements alambiqués au début des années 1830, affirme au commencement de 1846: "Soyons bien persuadés que ce qu'il y a de plus menacé, de menacé avant tout pour nous, ce n'est pas la liberté politique, qui

67 Voir dans Le Canadien du 30 septembre 1842, le discours qu'il prononça à la Chambre d'assemblée le 13 septembre.

68 William Ormsby, The Emergence of the Federal Concept in Canada 1839-1845 (Toronto, 1969).

69 Etienne Parent, Le Canadien, 28 septembre 1842. C'est, avant la lettre, ce qu'écrira Garneau dix ans plus tard dans la brève conclusion de la deuxième édition de son Histoire du Canada: "... Que les Canadiens soient fidèles à eux-mêmes; qu'ils soient sages et persévérants, qu'ils ne se laissent point emporter par le brillant des nouveautés sociales ou politiques... C'est aux grands peuples à essayer les nouvelles théories. Ils peuvent se donner des libertés dans leurs orbites assez spacieuses. Pour nous, une partie de notre force vient de nos traditions; ne nous en éloignons ou ne les changeons que graduellement... notre sagesse et notre ferme union adouciront beaucoup les difficultés de notre situation, et en excitant leur intérêt rendront notre cause plus sainte aux yeux des nations." F.-X. Garneau, Histoire $d u$ Canada depuis sa découverte jusqu'à nos jours, seconde édition corrigée et augmentée (Québec, John Lovell, 1852), III: 401-402.

70 Jacques Monet, s.j., The Last Cannon Shot. A Study of FrenchCanadian Nationalism 1837-1850 (Toronto, 1969). (Montréal, 1942). 
est pour ainsi dire indigène à ce continent, mais bien notre nationalité." ${ }^{2}$ Parent veut ici annihiler trop rapidement l'antinomie de la nation et de la démocratie. En voulant détourner la jeunesse des audaces politiques qui ne seraient pas permises aux Canadiens français, Parent ne peut accréditer qu'elle jouit déjà de "la liberté politique" et que ce qui importe c'est "avant tout" la consolidation de la nationalité. D'ailleurs, la régie intérieure du Canada-Est, que détiennent les chefs canadiens-français, est rognée par la pression exercée sur eux par la majorité économique britannique de cette section du Canada-Uni, et seule, elle ne peut leur permettre que de maintenir la nationalité canadienne-française.

A la fin de la même année, il donne de nouveau à croire à ses auditeurs qu'ils possèdent la liberté politique: “Au prix des longs et rudes travaux de vos aînés, vous voilà entrés dans la terre promise." "73 Cependant, l'année suivante, il sentira le besoin d'apporter une correction à sa définition de la situation politique de ses compatriotes en attestant qu'ils n'ont pas encore accédé à la royauté. Il les priera néanmoins de ne pas rêver et de se conduire en bon sujets:

L'on ne saurait trop répéter aux peuples, en travail d'émancipation politique, qu'il ne suffit pas, pour vouloir une chose, qu'elle soit bonne, juste et raisonnable en elle-même; mais qu'il faut en outre qu'elle soit possible sans déchirement, sans entraîner de ces folles luttes politiques, qui ne servent qu'à retarder les progrès de la liberté, en jetant les peuples dans le découragement. Puis il se trouve quelquefois des peuples dans une position toute particulière, à qui la prudence ne permet pas d'attendre, et pour qui, comme dit Lafontaine: Un tiens vaux [sic] mieux que deux tu l'auras. ${ }^{74}$

\section{L'IDÉE DE FÉDÉRATION MONDIALE}

Satisfait du caractère fédéral de l'Union, ${ }^{75}$ même si la double majorité, notion dont il s'était fait le propagateur dans Le Cana-

72 Etienne Parent, "L'industrie considérée comme moyen de conserver la nationalité canadienne-française" (1846), Le Répertoire National (4 vol., Montréal, 1848-1850), 4:7. A l'avenir, $R N$.

73 Etienne Parent, "Importance de l'étude de l'économie politique" (1846), $R N, 4: 33$.

74 Etienne Parent, "Du travail chez l'homme”, $R N, 4: 57$.

75 Il dira même le 7 février 1852: "A l'heure qu'il est cette unité [d'action politique] fait le désespoir de nos adversaires politiques, qui voient que, grâce à elle, nous nous sommes fait une arme de cette même union des Canadas, machine infernale qui a éclatté [sic] entre les mains de ses fabricateurs." De l'intelligence dans ses rapports avec la société (Québec, de l'imprimerie E.-R. Fréchette, 1852), 50. Parent répète ici ce que LaFontaine 
dien du 18 novembre 1844, ne s'est pas inscrite dans la pratique, et nourri des écrits socialistes dans lesquels "une sympathie naturelle" 76 tend à rapprocher socialisme utopique et fédéralisme romantique, Parent en vient à alléguer que la "national self determination" est une billevesée. Il estime que grâce à "la doctrine du libre échange... la liberté fera le tour du monde" ${ }^{77}$. Entrevoyant la formation prochaine d'une fédération mondiale, il demande:

... qu'y aurait-il donc de si absurde dans la prévision que les peuples se réuniront un jour, en congrès général, pour travailler de concert à la régénération de l'espèce humaine ? On a bien vu les rois tenir des congrès pour s'entendre sur les moyens de maintenir les peuples sous le joug, pourquoi les peuples n'en feraient-ils pas autant dans leur intérêt commun ? 78

Persuadé qu'ils “travailleront à répandre les bienfaits de la liberté, une fois qu'ils l'auront fermement établie chez eux",79 Parent suppose que "ceux qui seront engagés dans cette noble propagande aimeront, chercheront à coordonner, à concentrer leurs efforts afin d'en augmenter la puissance et l'efficacité. Que ce soit à Rome, à Londres, à Paris ou à Washington, les peuples auront un jour leur congrès", ${ }^{80}$ prédit-il. Il croit en déce-

disait quatre mois auparavant. Lors d'un banquet donné en son honneur à Montréal le 1er octobre 1851, quatre jours après l'annonce de sa démission à Elgin, LaFontaine expliqua: "... the Union was regarded by its authors as an agent with which to annihilate French Canadians. I regard the intention of this measure in that light; but after having carefully examined the rod by which it was intended to destroy my countrymen, I beseeched some of the most influential among them to permit me to use it, to save those whom it was unjustly designed to punish - to place my countrymen in a better position than they had occupied before. I saw that this measure enclosed in itself the means by which the people could obtain that control upon the Government to which they have a just claim; and the establishment in Canada of Responsible Government..." Sir Arthur G. Doughty, ed., The Elgin-Grey Papers 1846-1852 (Ottawa, 1937), 3:903. Tous deux démontrent clairement qu'ils ont bien assimilé les leçons de pancanadianisme que Francis Hincks leur donna en 1839-1840 et qu'ils jouent les Cyranos avec brio. Le "Responsible Government" à l'obtention duquel ils ont travaillé est détenu par la majorité britannique dans le Canada-Uni et sur cette scène, dans son rôle de jeune premier, LaFontaine ne fait qu'interpréter le scénario écrit par les Britanniques.

76 Claude Corbo, "Socialisme et fédéralisme au XIXe siècle: SaintSimon, Proudhon, Bakounine", dans Fédéralisme et Nations (Montréal, 1971), 83.

77 Etienne Parent, “Du travail chez l'homme” (1847), RN, 4:72-73.

78 Ibid., $4: 73$.

79 Ibid., $4: 73$.

80 Ibid., $4: 74$. 
ler un signe avant-coureur: "Eh! voyez donc ces sympathies politiques qui ne connaissent plus de frontières, qui s'élancent au-delà des océans comme autant de fils dont se formera la chaîne qui doit un jour lier les peuples libres dans une sainte et fraternelle union." ${ }^{81}$ Il avise qu'on ne s'enquerra plus si une nation possède son Etat mais qu'on cherchera plutôt à savoir si elle favorise la liberté à l'échelle planétaire:

Les distinctions nationales perdent leur ancienne signification; encore quelque temps, et il n'y aura plus, à proprement parler, d'anglais, de français, d'allemands et d'américains; il n'y aurait [sic] plus que des hommes progressifs ou rétrogrades, des égoïstes ou des libéraux. On ne s'informera plus si tel homme parle cette langue ou cette autre, mais seulement si ses paroles et ses discours sont ceux. d'un homme libre. 82

Parent précise le but des nouvelles sociétés: "il s'agira de la régénération de l'humanité entière" .83 Puis, prévoyant la disparition de la face du globe pour "les peuples innombrables qui ne sont pas encore en voie de régénération", 84 il invite alors ses compatriotes à jouer leur rôle "dans le grand drame du monde". ${ }^{85}$ Enfin, après s'être dit "assuré que ce rôle ne fera pas rougir les mânes de nos pères" ${ }^{86}$, il fait appel à la vocation des peuples [inversement proportionnelle à leur grandeur] :

Nous surtout, Canadiens-Français, issus d'une race éminemment chevaleresque, qui sait si nous ne sommes pas destinés à installer dans la politique de ce continent cet esprit de bienveillance et de générosité, sans lequel la société humaine ne saurait atteindre la plus noble de ses fins, le progrès moral de notre espèce ? 87

Cinq ans plus tard, la foi d'Etienne Parent dans la formation prochaine d'une fédération mondiale s'est attiédie mais sa conviction des bienfaits du commerce international libre demeure aussi vivace:

S'il est possible, ce dont je doute, s'il est désirable, ce dont je doute encore davantage, qu'il y ait sur notre terre une domination universelle, ou l'unité gouvernementale, que ce soit sous les auspices du génie commercial; car avec les idées plus éclairées qui se répandent en fait d'économie politique, ce sera le régime le plus favorable au bien-être de l'humanité...

81 Ibid., $4: 74$.

82 Ibid., $4: 74$.

83 Ibid., $4: 75$.

84 Ibid., $4: 76$.

85 Ibid., 4:77.

86 Ibid., 4:77.

87 Ibid., $4: 78$. 


\begin{abstract}
Encore une fois, laissez faire le commerce, et vous verrez comme il saura remplir la mission providentielle dont il est chargé, de rápprocher les hommes, de les faire se connaître, se communiquer leurs idées, échanger leurs richesses, et par là de réformer la grande famille humaine dispersée à la Tour de Babel. Il s'est fait et se fait encore de beaux rêves sur la fraternité humaine, sur les moyens d'arriver à la fraternisation universelle. Le plus sûr de ces moyens, nous l'avons dans le commerce, dans le commerce libre...88
\end{abstract}

Voilà comment Etienne Parent résolvait, vers 1850, "tout le problème, qui était celui de l'Etat, de la nation et du fédéralisme" 89 au moment où les "British-Americans" rejetaient l'idée d'annexion aux Etats-Unis quelques mois seulement après en avoir prôné l'incarnation et acceptaient de payer le coût social de la construction d'une économie nationale. ${ }^{90}$

\title{
LES PREMIERS CANADIENS FRANÇAIS : \\ DES PUPILLES DE VIEILLE SOUCHE
}

Comment expliquer ce point de chute? Les Canadiens français développaient depuis longtemps des dispositions à se transformer en pupilles. Lors de la période d'agitation verbale dans les treize colonies, les "nouveaux sujets" craignant d'être engouffrés dans un grand ensemble américain républicain se cherchent un défenseur. Ils avancent: “... S'il est un moyen d'empêcher, ou du moins d'éloigner cette révolution, ce ne peut être que de favoriser tout ce qui peut entretenir une diversité d'opinions, de langage, de mœurs et d'intérêt entre le Canada et la Nouvelle-Angleterre." 91 L'Acte de Québec reconnaît, en 1774, l'existence d'une population française dans la grande "Province of Quebec" mais il ne peut "empểcher ou éloigner" la révolution américaine. Une fois celle-ci réussie, d"anciens sujets" immigrés dans la colonie "soutiennent qu'il faut en autant que possible, tenir les nationaux de cette province à l'écart des autres colons et sans relations avec ceux-ci, afin de servir de rempart

88 Etienne Parent, De l'importance et des devoirs du commerce (Québec, 1852), 11 et 12 .

89 Jean-Paul Bernard, Les Rouges-Libéralisme Nationalisme et Anticléricalisme au milieu du XIXe siècle (Montréal, 1971), 51.

90 Pour insérer "L'idée de fédération chez Etienne Parent" dans le contexte "British North American", on peut consulter avec profit JeanCharles Bonenfant, "Les projets théoriques de fédéralisme canadien", Les Cahiers des Dix, no 29 (Montréal, 1964), 71-87 et L.F.S. Upton, "The Idea of Confederation, 1754-1858", dans W.L. Morton, éd., Le Bouclier d'Achille (Toronto, 1968), 184-207.

91 Mémoire cité dans F.-X. Garneau, op. cit., 1:XXI. 
solide entre nos établissements et les Etats-Unis". ${ }^{92}$ Cette idée de peuple-frontière sera par la suite rappelée par D.-B. Viger, ${ }^{93}$ Pierre Bédard, ${ }^{94}$ L.-J. Papineau ${ }^{95}$ et Toussaint Pothier ${ }^{96}$ avant d'être rebattue par Parent.

Maîtres incontestés d'une Chambre d'assemblée, les Canadiens, au début des années 1830, aspirent à l'indépendance. Craignant de ne pouvoir la maintenir devant la croissance tentaculaire des Etats-Unis, Parent, pour sa part, se cherchera un protecteur dans une fédération canadienne. La perte des grands pouvoirs politiques n'est pas un inconvénient majeur. Papineau, Elzéar Bédard et Morin, pour leur part, voient dans un futur Bas-Canada indépendant un allié de la Grande-Bretagne, ${ }^{97}$ alliance dans laquelle le gros protégerait le petit. Avant d'obtenir cette émancipation coloniale, les Canadiens réclament la quasiindépendance sous l'aile protectrice de l'Angleterre. Pour la lui arracher, ils veulent former un front uni avec les réformistes du Haut-Canada. ${ }^{98}$ Cette liaison d'amitié qu'ils tentent d'établir amènera Morin à confier à Hincks que lui et ses collègues se présenteront à la première session de la législature unie pour y revendiquer leurs droits "as Canadians".99 Bientôt maîtres de l'administration de leur section du Canada-Uni, ils se flattent d'être devenus des Canadiens français. Même, au cours de son duel oratoire avec Papineau en 1849, LaF'ontaine prétendra jouir de l'égalité politique avec les "British-Americans":

...si l'Irlande avait eu avec l'Angleterre cette partie égale dans la représentation, comme le Bas-Canada la possède aujourd'hui avec le Haut-Canada, l'Irlande n'aurait pas été asservie comme elle ne l'a que trop été malheureusement; ses

92 Lettre de Hugh Finlay à Sir Evan Nepean, 13 février 1787, Documents relatifs $\grave{a}$ l'histoire constitutionnelle du Canada 1759-1791, Adam Shortt et Arthur G. Doughty, éd., (Ottawa, 1921), 2:830.

93 Voir les Considérations ... par un Canadien, M.P.P. (1809), brochure citée par John Hare et Jean-Pierre Wallot, Confrontations Ideas in Conflict (Editions du Boréal Express, 1970), 27-58.

94 Voir le "Mémoire... soumis à la considération de Milord Bathurst, ministre d'état pour les colonies" (1814), W.P.M. Kennedy, op. cit., 282-287.

95 Voir les "Observations de MM. Papineau et Neilson sur le projet de réunir les législatures du Haut et du Bas-Canada", dans Appendices aux Journaux de la Chambre d'Assemblée du Bas-Canada (1825), appendice K. 96 Mémoire de Toussaint Pothier à l'égard de la situation politique du Canada (1829), Rapport sur les Archives canadiennes (1913): 92-103.

${ }^{97}$ Les "92 Résolutions", résolution 21, Journaux de la Chambre d'assemblée du Bas-Canada (session de 1834), 43:314.

98 Etienne Parent, 13 mai 1833.

99 Lettre d'Augustin-Norbert Morin à Francis Hincks, Québec, 8 mai 1841, Douglas Brymner, Report on Canadian Archives (Ottawa, 1884), 170 
habitants ne périraient pas de famine et de misère dans leurs demeures et sur la voie publique; ils auraient eu, dans leurs co-sujets anglais, non des maîtres, mais des égaux ...100

Qui pouvait alors faire comprendre à LaFontaine que les Canadiens français de 1850 sont annexés du point de vue politique à la nation "British-American" majoritaire, vivent par procuration de la richesse d'autrui et peuvent tout juste assurer leur survivance culturelle.

Le "Grand Homme" lui-même ne pouvait démontrer l'impossibilité pour les Canadiens français d'être vraiment maîtres dans un Canada-Est qui ne serait pas séparé politiquement. En 1834, dans sa demande d'un nouveau statut colonial, il laissait "une autorité de surveillance dans le gouvernement impérial, pour décider de la paix et de la guerre et des relations de commerce avec l'étranger" 101. Cinq ans plus tard, en réponse au rapport Durham, il se prononce en faveur de l'indépendance du Bas-Canada, mais il se montre favorable aussi à l'entrée du Bas-Canada dans la grande fédération américaine "avec individualité pour chaque Etat souverain, sous la protection du Congrès, qui ne pouvait être tyran n'ayant ni sujets, ni colonies, et ne possédant d'attributions que dans les questions de paix ou de guerre avec l'étranger et de commerce extérieur..." 102 Comme pour nos autres premières "Gloires nationales", la recherche omniprésente d'une autorité tutélaire amène Papineau à confondre "self government" et "national self determination". Et, c'est à ce niveau étale que les chefs canadiens-français vers 1850 se figent.

100 L.-H. LaFontaine, Chambre d'assemblée du Canada-Uni, 23 janvier 1849, Thomas Chapais, Cours d'histoire du Canada (Québec, 1933), 6:304. 101 Discours de Papineau "aux libres et indépendants électeurs du quartier ouest de Montréal", La Minerve, 4 décembre 1834, cité par Fernand Ouellet, Papineau - textes choisis (PUL, Québec, 1970), 65.

102 Louis-Joseph Papineau, Histoire de l'insurrection du Canada (Montréal, 1963), 16. 